\title{
Research on high frequency arc transducer array directivity
}

\author{
LIU Zhen-jun
}

Science and Technology on Underwater Acoustic Antagonizing Laboratory, Shanghai, China

Izjun@smmail.cn

Keywords: high frequency; arc transducer array; wide beam; directivity

\begin{abstract}
High frequency arc transducer array is mainly used in multi-beam image sonar, transmitting wide beam waves. The directivity diagram of the high frequency arc transducer is calculated based on the theoretical formula and the directivity characteristics are analyzed. Laboratory verification is conducted for the high frequency arc transducer array that uses 1-3 piezoelectric composite materials.
\end{abstract}

\section{Introduction}

High frequency arc transducer array is often used in ocean engineering, the underwater archaeology, the salvage and rescue and other underwater operations and image sonar in underwater security ${ }^{[1-3]}$. High frequency arc transducer array is characterized by wide beam directivity, and thus can cover the whole scope of vision with a single transmitting beam. High resolution detection can be achieved through the receiving array beam rotation. Multi-beam image sonar generally works in hundreds of $\mathrm{kHz}$ frequency spectrum, in order to achieve high resolution in small volume weight. The research on the directional characteristic of the arc transducer array workig in hundreds of $\mathrm{kHz}$ frequency is helpful to design high frequency arc transducer array with large wave beam angle, small fluctuation and easy manufacturing technics. This article calculates the directivity diagram of the high frequency arc transducer through the theoretical formula and explicits its directivity characteristics.

\section{Basic directivity characteristics of the high frequency arc transducer}

As described in this article, the arc transducer array has a cylinder radiating surface and its cross section is a circular arc. It is composed of several basic element close, and the primitive vibration is plane piston vibration. The horizontal directivity of the arc transducer is calculated based on the arc directivity formula, as shown in formula (1).

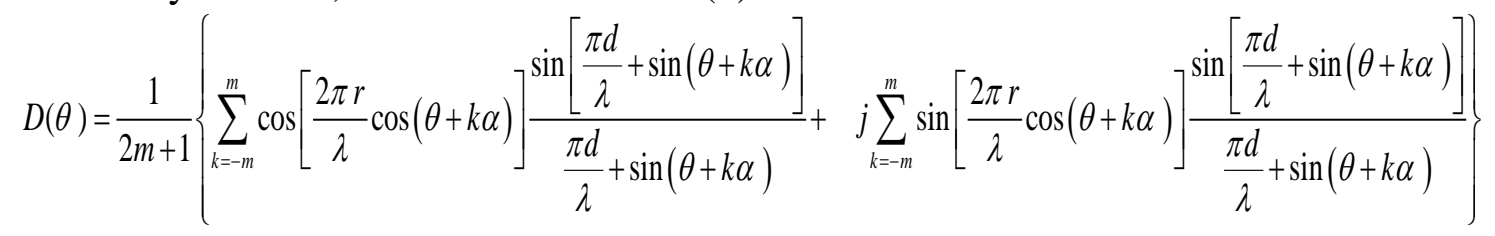

In the formula,

$2 \mathrm{~m}+1$ : Number of primitives

$\lambda$ : The wavelength

$\mathrm{d}$ : Center distance of the primitives

r: Radius of the arc array

$\alpha$ :Central Angle of a single primitive

Formula (1) shows that the horizontal directivity is mainly determined by the wavelength (frequency), the radiating surface radius and the corresponding parameters such as central Angle. Figure 1 shows the $500 \mathrm{kHz}$ directivity diagram calculated by formua (1), assuming 101 primitives, 101 degrees of the radiation surface central angle and a radius of $100 \mathrm{~mm}$. All directivity diagrams are normalized linear graph in this paper. 


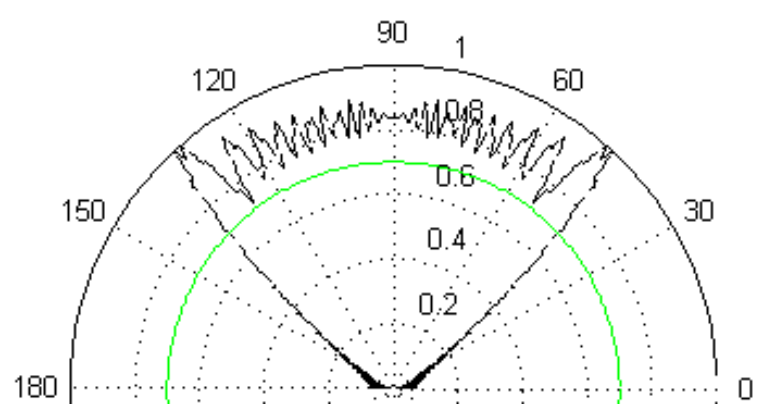

Fig.1 Typical directivity diagram of the arc array

Figure 1 shows that the arc transducer array has obvious directivity, and has the following characteristics: wide beam, beam width close to the transducer arc radiation surface central Angle, the maximum value of the main beam is in the edges on both sides, there is continuous fluctuation within the transmitting beam and the fluctuation degree increases gradually from the center to both sides; there is a series of side lobe outside the main beam and sidelobe's open Angle is small, and the amplitude decreases gradually so that the main beam edge becomes serrated and declines quickly.

\section{Frequency response characteristics of directivity}

\section{Calculation and analysis}

Take the high frequency arc transducer array with a diameter of $170 \mathrm{~mm}, 125$ primitives, and a central Angle of 125 degrees as an example; that is, $r=85 \mathrm{~mm}, \mathrm{~d}=1.5 \mathrm{~mm}, \mathrm{a}=1^{\circ}$ Calculate the directivity of the transducer at $100 \mathrm{kHz}$ to $800 \mathrm{kHz}$ (that is, $\lambda$ ranges from $2 \mathrm{~mm}$ to $15 \mathrm{~mm}$ ) with the bandwidth of 4 octave, and analyze the characterisitics and rules of the high frequency arc transducer array'directivity, which changes with the frequency. After the preceding parameters are placed in the formua (1), the directivity diagram is calculated, as shown in figure 2.

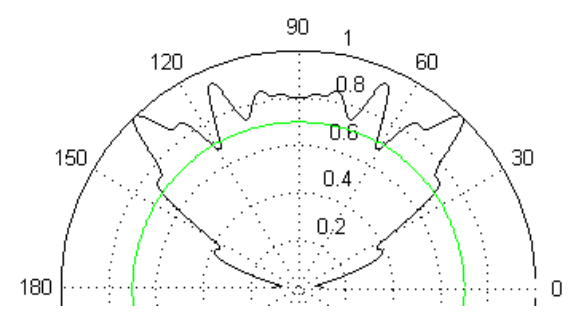

a. $100 \mathrm{kHz}$

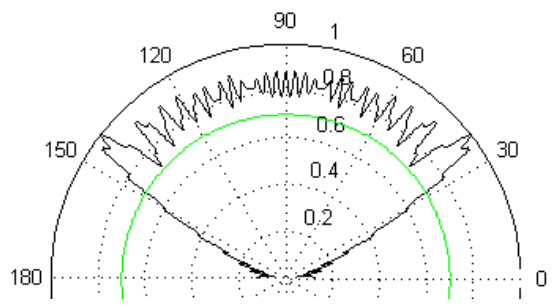

c. $400 \mathrm{kHz}$

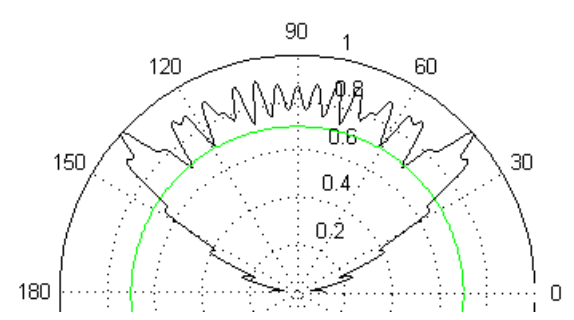

b. $200 \mathrm{kHz}$

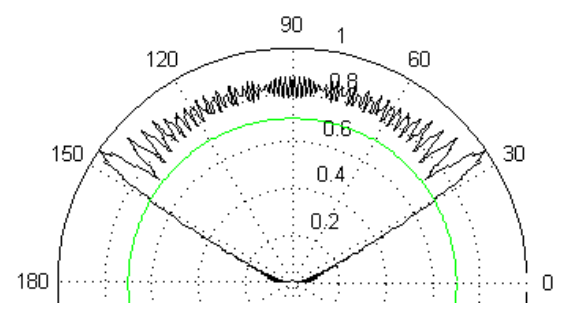

d. $800 \mathrm{kHz}$

Fig.2 Frequency response characteristics of directivity

It can be seen from figure 2, at a diameter of $170 \mathrm{~mm}, 125$ primitives, $125^{\circ}$ of central Angle, arc transducer array in $100 \mathrm{kHz}$ to $800 \mathrm{kHz}$ features arc array orientation, large beam opening Angle, and continuous fluctuation within the transmitting beam. With the increase of frequency, beam Angle increases slowly, even can be thought of remaining unchanged; And the number of fluctuations in the beam is significantly increased, the fluctuation degree decreases slightly on the contrary. Figure 3 is a curved line array and array directivity open Angle frequency response curve 
contrast. It indicates when frequency is low, the arc array degrades to linear array when the differece between a circular arc peripheral primitive and the centre primitive tangent chord is less than $1 / 2 \lambda$. When the frequency is higher and the differece between a circular arc peripheral primitive and the centre primitive tangent chord is greater than $\lambda$, a typical circular array is formed. Its beam open Angle gradually increases and approximates to circular central Angle as the frequency increases.

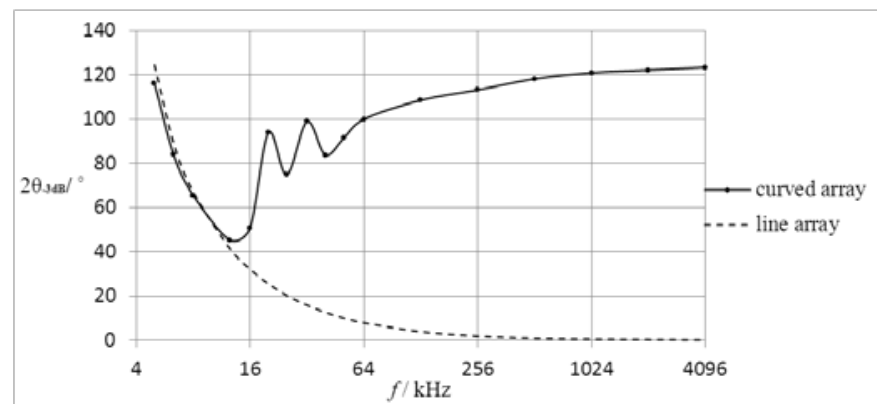

Fig.3 Contrast of Arc array and linear array directivity open Angle

\section{Experimental verification}

We use 1-3 type piezoelectric composite material and develope a high frequency arc transducer array with wide beam transmission. The transducer array resonant frequency is $530 \mathrm{kHz}$, its $1-3$ piezoelectric composites has a diameter of $170 \mathrm{~mm}$, a height of $8 \mathrm{~mm}, 105^{\circ}$ central Angle. Figure 4 is the transducer array photograph.

Performance tests are conducted in free-field pool for the arc transducer array. Test results show that the horizontal directivity diagram (figure 5) has the characteristics of typical open Angle curved transducer array: at $315 \mathrm{kHz}$ to $700 \mathrm{kHz}$ frequency within the open Angle of emission (horizontal open Angle of $100^{\circ}$ ), the main beam is obvious and there is continuous fluctuation within the main beam. Measured directivity, besides certain directions with fluctuation of more than $3 \mathrm{db}$ within the main beam due to factors such as primitive consistency, fully meets the directivity and frequency response characteristics in formula (1).

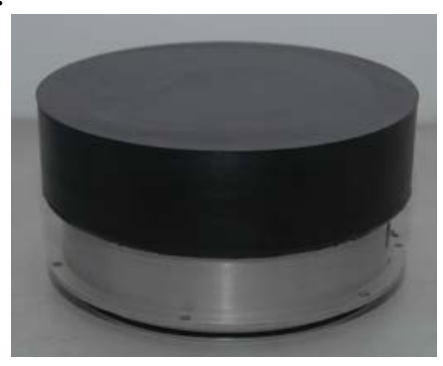

Fig.4 Photo of the transducer array

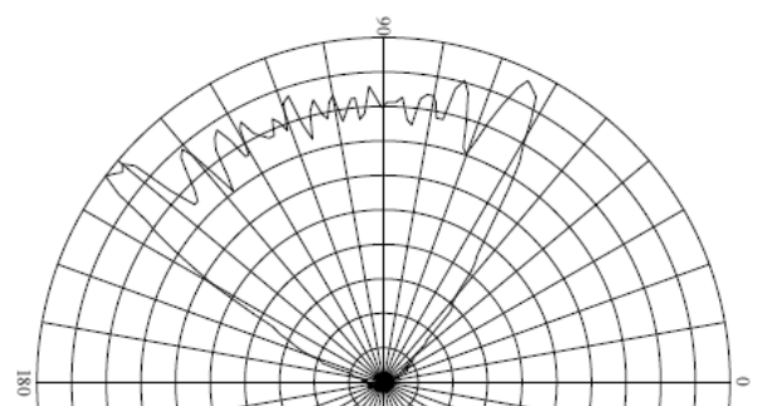

a. $315 \mathrm{kHz}$

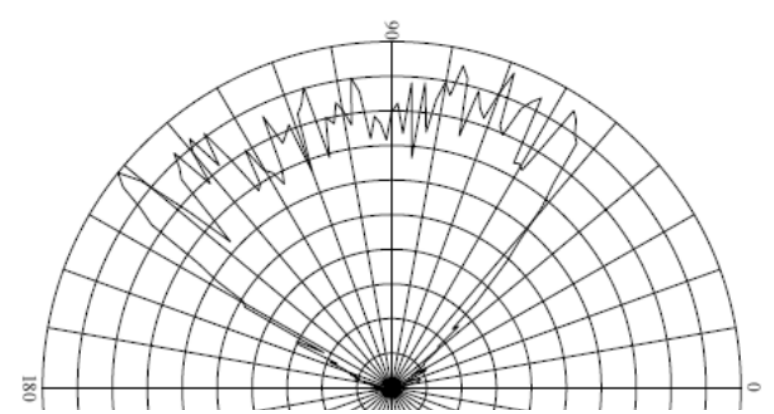

b. $500 \mathrm{kHz}$ 


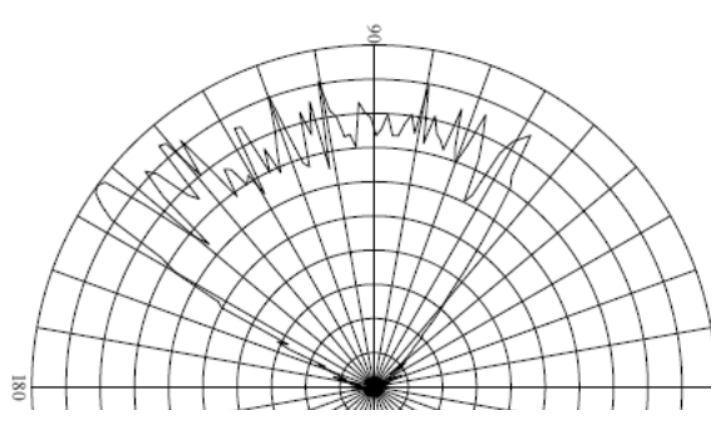

c. $600 \mathrm{kHz}$

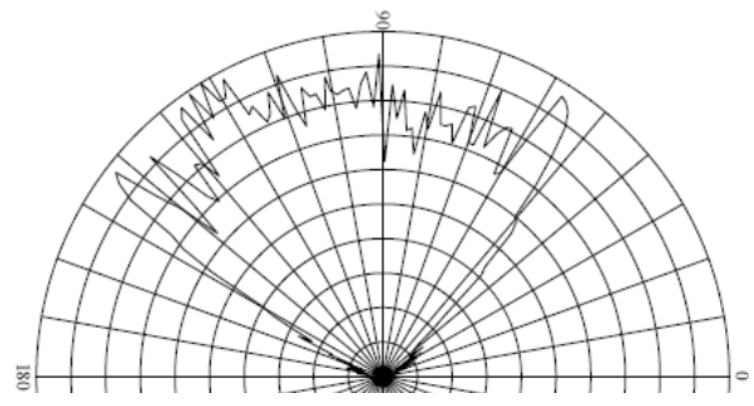

d. $700 \mathrm{kHz}$

Fig.5 The measured directivity diagrams for different frequencies

\section{The influcence of primitive quantity on directivity}

The high frequency arc transducer array with a diameter of $170 \mathrm{~mm}$ and a central Angle is $125^{\circ}$ is still used as an example. When the frequency is $500 \mathrm{kHz}$, and calculated value from Formula (1) at different primitive number when $\mathrm{m}=4-1800$, arc respectively composed of 9-3601 primitives directed graph is as follows (figure 6). When the number of primitives is small, the scale of the individual primitives is large, primitive has a continuous linear array directivity, the primitive directivity open Angle is less than the corresponding central Angle, the directivity associations between adjacent primitives are small. Therefore, after the arc array directivity performance for each primitive in their own position to create a transmittig beam, just due to the interference effect of the other primitive radiation beam at the top, it is not smooth, there is strong fluctuation within the array beam. As the number of primitives increases, the directivity of a single primitive becomes increasingly big, and the primitive associations become strong, gradually forming a typical arc array directivity. When the primitive directivity opening Angle is three times greater than the central Angle, it becomes stable.

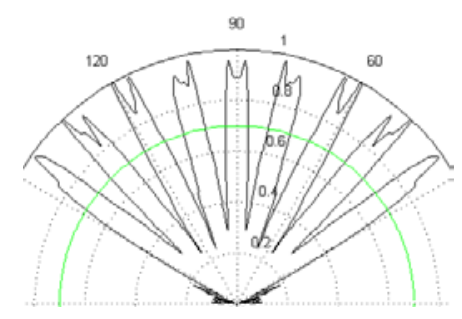

a. 9 primitives

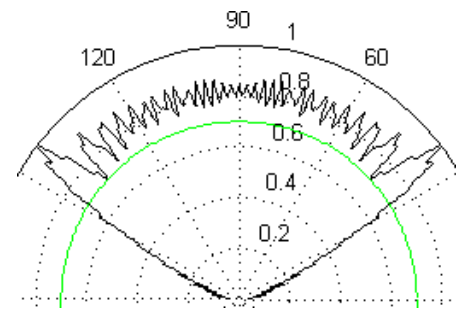

d. 35 primitives

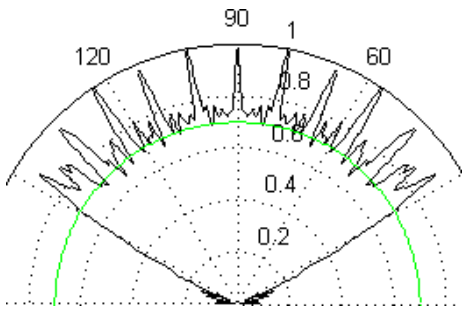

b. 13 primitives

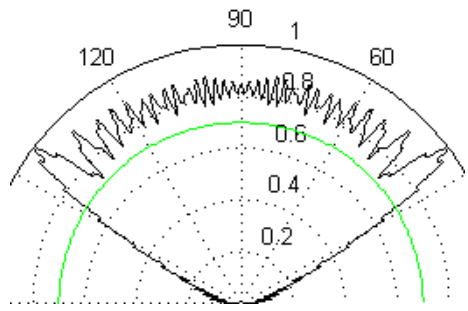

e. 361 primitives

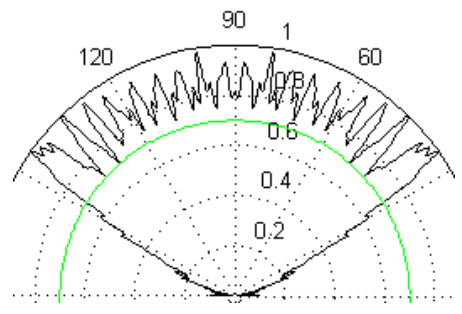

c. 21 primitives

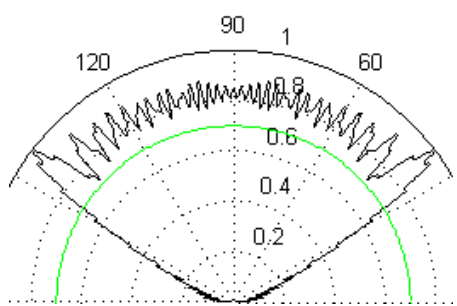

f. 3601 primitives

Fig.6 Impact of primitive number on directivity

\section{Influence of the array diameter}

The high frequency arc transducer array with a diameter of $170 \mathrm{~mm}$ and a central Angle is $125^{\circ}$ is still used as an example. When the frequency is $500 \mathrm{KHZ}$ and inter-primitive distance is $1 \mathrm{~mm}$, the directivity diagram for $r=1-10000-\mathrm{mm}$ is calculated as follows (figure 7). When the diameter is small, the distance between the circular arc edge primitive and tangent arc center is smaller than the wavelength, and the arc array directivity is linear array directivity; with the increase of diameter, 
it is gradually becoming a typical arc array directivity, and fluctuation quantity within the beam increases significantly and the fluctuation degree becomes increasingly small, and open Angle of the beam is approaching the central Angle.

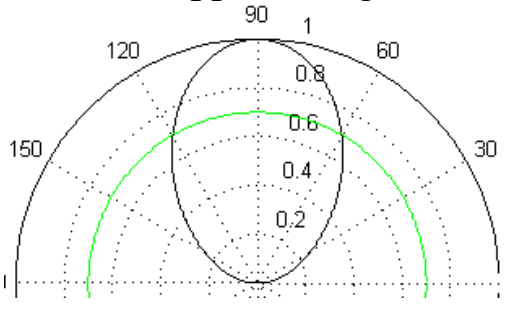

a. $\mathrm{r} / 1.5 \mathrm{~mm}$

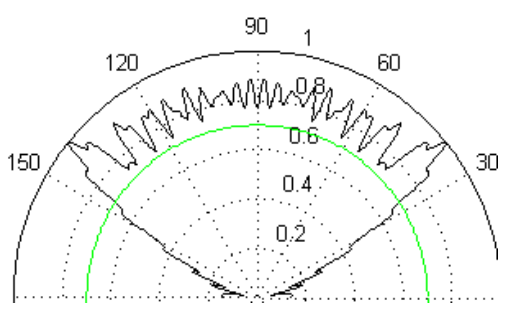

d. $\mathrm{r} / 50 \mathrm{~mm}$

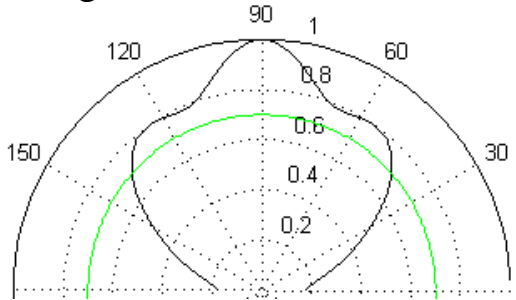

b. $r / 3 m m$

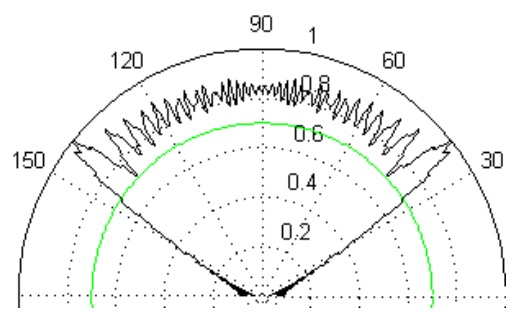

e. $\mathrm{r} / 100 \mathrm{~mm}$

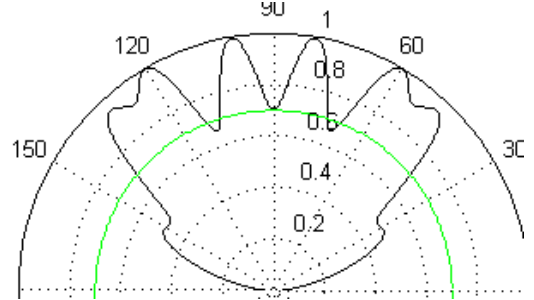

c. $\mathrm{r} / 10 \mathrm{~mm}$

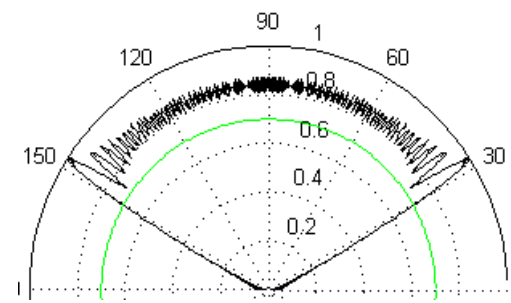

f. $r / 250 m m$

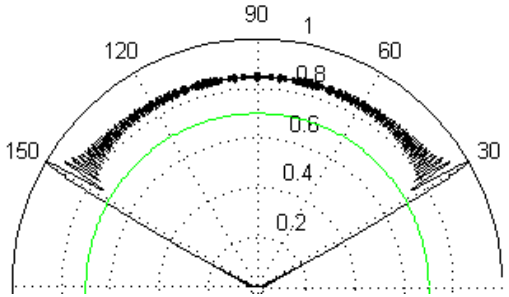

h. $\mathrm{r} / 1000 \mathrm{~mm}$

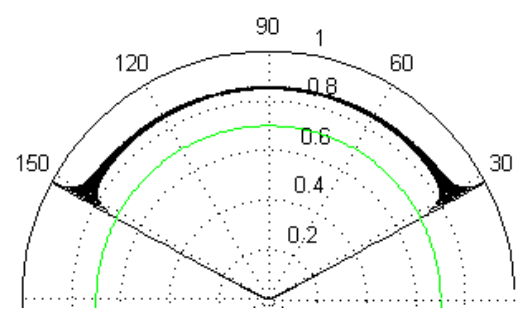

i. $\mathrm{r} / 10000 \mathrm{~mm}$

Fig.7 The influence of curved transducer array diameter on directivity

\section{Summary}

The high frequency arc transducer array directivity characteristics are calculated and analyzed according to the directivity formula for the high frequency arc transducer array, including the influences of parameters like frequency, the number of primitives and diameter on the directivity.

\section{Reference}

[1] C.Desilets. Composite Curved Linear Array for Sonar Imaging. IEEE ULTRASONICS SYMPOSIUM. 1997, 901-906.

[2] ZHOU Li-sheng, HU Qing. Summarization of underwater acoustic projector technologies. Journal of Harbin Engineering University. 2010（7） . 932-937.

[3] ZHONG Lin-jian, TANG Jun, ZHONG Qin-qin. Research of high frequency sphere coronal transducer. Acoustics andelectronic engineering. 2011（3） . 22-25. 